\title{
Criminologie
}

\section{Une illustration de la peur concrète : le cas des victimes}

\section{Micheline Baril}

Volume 16, numéro 1, 1983

La peur du crime

URI : https://id.erudit.org/iderudit/017170ar

DOI : https://doi.org/10.7202/017170ar

Aller au sommaire du numéro

Éditeur(s)

Les Presses de l'Université de Montréal

ISSN

0316-0041 (imprimé)

1492-1367 (numérique)

Découvrir la revue

Citer cet article

Baril, M. (1983). Une illustration de la peur concrète : le cas des victimes.

Criminologie, 16(1), 31-49. https://doi.org/10.7202/017170ar d'utilisation que vous pouvez consulter en ligne.

https://apropos.erudit.org/fr/usagers/politique-dutilisation/ 
UNE ILLUSTRATION DE LA PEUR CONCRÈTE : LE CAS

DES VICTIMES

Micheline Baril*

La peur est une mauvaise gardienne; elle joue son jeu tragique en marge des risques réels, et souvent à contresens.

Alain

Parfois contradictoires, souvent étranges, les résultats des études sur la peur du crime ne cessent d'étonner. On y apprend, par exemple, que les gens qui ont le plus peur n'adoptent pas nécessairement plus de mesures ou de comportements de prévention que ceux qui manifestent moins d'inquiétude. La plupart des travaux rapportent que les personnes qui craignent le plus le crime et qui font un grand usage de mesures préventives ne sont pas celles qui sont le plus exposées à une agression criminelle. Ainsi, bien que les femmes et les personnes âgées courrent relativement peu de risques de devenir la cible d'activités criminelles, elles manifestent pourtant le plus de peur (Hindelang, Gottfredson et Garofalo 1978, Skogan et Maxfield 1981, Rigier 1982).

Il est suggéré aussi que le degré d'information et notamment l'influence des mass média auraient plus d'impact sur la peur du crime que les expériences personnelles (Brillon, Louis-Guérin et Lamarche 1982, Van Kijk 1978). D'ailleurs, la plupart des sondages dans lesquels cette relation a été étudiée concluent que l'expérience de victimisation a peu ou pas d'impact sur le degré de crainte ressentie (voir entres autres Hindelang, Gottfredson et Garofalo 1978, Garofalo 1979, Genn 1976). On aurait pourtant pu s'attendre à ce qu'elle soit un des facteurs les plus déterminants. Il reste à voir si ces résultats reflètent la réalité ou bien des problèmes méthodologiques de mesure.

Une êtude qualitative réalisée, il y a quelques années, au moyen d'entrevues non structurées, révélait que les Québécois qui n'avaient jamais subi de crimes violents manifestaient très peu de peur à l'égard de la violence alors que, chez les victimes, cette peur était intense et faisait partie du quotidien (Baril 1977). Il faut noter cependant que dix victimes seulement avaient été interrogées.

Depuis, d'autres recherches ont été réalisées au Québec auprès de victimes d'actes criminels. Leur objectif n'était pas d'étudier la peur du crime en particulier mais de mieux connaître les expériences,

* Chargé d'enseignement, École de criminologie, Université de Montréal. 
les réactions et les attitudes des personnes victimisées. Nous analyserons ici les discours recueillis dans deux de ces études. La première a été faite en 1977 auprès de quarante petits commerçants victimes de vol à main armée (Grenier, Manseau 1077). Dans la seconde, en cours, nous avons rencontré soixante-cinq victimes des crimes suivants : tentative d'homicide, voie de fait, vol qualifié, agression sexuelle, enlèvement et séquestration, cambriolage, de même que des proches de victimes d'homicide.

Presque toutes les victimes d'actes violents ont parlé spontanément de la peur qu'elles avaient éprouvée au moment de l'événement criminel ou après. La peur est d'ailleurs, généralement, l'émotion qui transparait le plus clairement dans leurs propos et dans leurs comportements. Plusieurs ont déménagé, changé d'emploi ou de numéro de téléphone, depuis l'agression. Elles sont réticentes, souvent, à accorder une entrevue.

$\mathrm{Au}$ début, quand vous m'avez appelée, je ne voulais pas vous rencontrer parce que juste le fait de vous en parler, je revis tout ça bien facilement. J'avais peur continuellement pendant un an. J'ai accepté pour vous aider si ça peut aider à quelque chose.

Après avoir regardé la nature, l'objet et les circonstances de la peur chez les victimes, nous verrons si des mesures de prévention et des attitudes d'intolérance à l'égard du crime et des criminels sont associées à cette peur. Nous proposerons aussi quelques hypothèses pour expliquer les faibles liens qu'on trouve entre la peur et les expériences de victimisation dans la plupart des études.

\section{LA PEUR COMME RÉACTION IMMEDIATE}

La violence provoque presque toujours une peur immédiate, ou même une panique, chez celui qui en fait l'objet. La surprise de l'attaque jointe à la menace, à la présence d'une arme ou aux coups, produit une vive émotion et la réalisation d'un danger pour sa vie ou pour son intégrité physique.

J'ai eu seulement le temps de réaliser que quelque chose n'était pas normal et c'est arrivé. En l'espace d'une seconde, j'étais face à face avec un couteau qui avait l'air bien vicieux. Je transpirais parce que je savais que j'étais fini. C'était un couteau avec une lame fine, solide, qui peut passer à travers le corps comme rien. Je me sentais très mal.

Dans presque tous les cas, leur vie n'était pas sérieusement menacée mais les victimes ont cru qu'elles allaient mourir. Il est vrai 
qu'en présence d'une arme ou d'agresseurs plus forts que soi, un danger est toujours présent.

C'est la mitraillette que j'ai vue, pas d'autre chose. Il n'y a pas de risques à prendre avec quelque chose comme ça dans la face, on sait jamais quand ça peut partir. Quand même ce serait par accident, des fois, où que le gars il est bien nerveux, puis le coup part.

Dans certains cas, surtout si le crime se déroule très vite, la réaction ne se fait sentir qu'une fois l'événement terminé.

C'est seulement après, que je me suis dit : c'était un hold up. Et là, j'ai bien pleuré, ça m'a portée à pleurer. Par la suite, c'est là que ça été, pratiquement deux heures, bien difficile. J'ai eu vraiment un choc pendant deux heures et l'autre caissière aussi. On sent ça dangereusement ; ils avaient des grosses carabines. Ça m’a marquée.

Parfois, la peur est paralysante, comme lorsque la victime ne réussit pas à obéir aux ordres qui lui sont donnés ; à d'autres moments, elle se traduit par des cris ou une agitation extrême; il arrive aussi qu'elle soit bien contrôlée.

J'ai gardé mon sang-froid parce que j'avais peur qu'il fasse le tour du comptoir puis qu'il m'assomme parce que j'avais rien que $20 \$$. Je me suis dit : il va trouver que j'en ai pas asssez, puis il va m'assommer.

Même lorsqu'il n'y a pas de contact avec l'agresseur, comme dans le cas du cambriolage, la peur peut être un élément important. Elle survient au moment de la découverte du vol.

La porte était restée ouverte. Là, je suis venue mal. C'était impossible parce que c'était en hiver. En entrant, j’ai réalisé et puis je suis restée figée. Dans un éclair, je me suis dit : ils sont peutêtre encore là. Je suis sortie sur la pointe des pieds, bien doucement, pour ne pas leur faire peur, au cas où, et après j'ai couru chez mon dépanneur.

La peur semble plus intense lorsque l'agresseur est inconnu ou masqué, lorsque les menaces proférées ne sont pas mises à exécution, et lorsqu'il s'agit d'une première victimisation. Il est très difficile cependant de tirer des conclusions sur les déterminants de la peur immédiate. Les exceptions sont trop nombreuses. On peut dire toutefois qu'une peur intense lors de la victimisation risque fort de se prolonger assez longuement en comportements d'évitement, en phobies, cauchemars, adoption de mesures de protection. Chez les victimes qui disent n'avoir ressenti aucune crainte au moment de la victimisation, il arrive 
parfois que des réactions de peur se développent après un certain temps.

J'ai bien pris ça sur le coup. Ça s'est bien passé. J'ai eu peur, une fraction de seconde, disons, quand il m'a pris par le bras pour me retourner de bord : mets les mains sur ton bureau, regarde le mur. J'ai dit : ça y est, il va m'assommer, c'est ça. J'ai pensé, il va me casser un gun derrière la tête. Non, il n'a rien fait. À ce moment-là, je me suis calmé plus. (...) Ça s'est bien passé, disons ça fait rien qu'une minute à peu près. C'est pas trop pire mais c'est une expérience que je ne voudrais pas repasser, de toute façon au cas où, des fois, ça se passe pas pareil. J'y pense tous les jours parce que s'il y avait une autre fois, peut-être que je ne m'en sortirais pas.

Il arrive que des personnes aient connaissance des événements sans voir ce qui se passe, soit qu'elles sont dans une autre pièce, soit qu'on les ait enfermées ou qu'on leur ait bandé les yeux. L'imagination aidant, elles s'attendent au pire et sont terrifiées. Pourtant, un sujet de l'échantillon nous dit : "Le fait de ne pas voir, c'est moins pire."

\section{UNE PEUR QUI PERSISTE}

La plupart des victimes de violence que nous avons rencontrées sont demeurées craintives et méfiantes après l'événement. Dans certains cas, nous les avons vues quelques années après l'agression et leurs craintes subsistaient toujours. Les seules personnes chez qui nous n'avons pas remarqué de séquelles sur ce point ont été des hommes victimes de cambriolage et quelques personnes qui avaient subi plusieurs vols à main armée à leur lieu de travail.

La méthode d'enquête utilisée ne permet pas d'estimer durant combien de temps persistent les symptômes d'une peur associée à la victimisation. À notre connaissance, aucune étude n'a systématiquement étudié cette conséquence chez divers types de victimes. On possède plus d'information dans le cas de l'agression sexuelle. Par exemple, une étude de Killpatrick, Resick et Veronen (1981), utilisant un groupe contrôle, est arrivée au résultat que les femmes qui avaient été violées éprouvaient significativement plus d'anxiété, de crainte et de méfiance que les sujets du groupe contrôle, un an après l'agression. Elles s'imposaient également plus de restriction dans leurs comportements et modes de vie. Les travaux sur les survivants des camps de concentration illustrent abondamment les séquelles à long terme, parfois indélébiles de la victimisation ; la peur est au nombre de ces séquelles (Eitinger 1964). 
La peur peut être généralisée ou porter sur des objets précis. Dans ce dernier cas, ce qu'on craint le plus souvent est de revivre le même événement. Il faut dire que des 105 personnes dont il est question ici, 68 avaient subi un vol qualifié, la plupart à leur lieu de travail. Leurs craintes reposaient donc sur des fondements très réels, d'autant plus que plusieurs d'entre elles étaient loin d'en être à leur première expérience. Les victimes redoutent une répétiton de la violence subie, certes, mais

Jusqu'ici, on a eu plus de peur que de mal. L'argent compte, on travaille assez fort pour la gagner mais je dis tout le temps que nos vies, c'est bien plus important. J'ai une fille de 17 ans et un garçon de 15 ans et s'il leur arrivait quelque chose... Ça, j'ai bien peur de ça. Mon doux, j'ai des frissons juste à y penser. Des fois, on se demande si on met la vie des enfants en danger ou si on ne serait pas mieux sur le bien-être et sans risques.

On constate la même attitude chez plusieurs victimes de voies de fait qui connaissaient leurs agressseurs. La récidive est alors possible ou probable et la victime, qu'elle essaie sérieusement ou non de s'en protéger, en est consciente. Des personnes qui ont subi un vol avec effraction expriment aussi cette crainte d'une répétition du crime, possiblement de la part des mêmes voleurs.

Ils sont venus une fois et ça a bien marché. Du butin, pas de problèmes, pas fait prendre. Ils connaissent la place, nos habitudes; on a beau essayé de changer. Comme les voleurs, à mon idée, c'est des lâches, ils vont revenir.

Dans les deux situations, on anticipe une escalade de la violence, on imagine les pires conséquences : "La prochaine fois, il me tuera "; "et si je surprenais le voleur pendant qu'il est encore dans ma maison? "Le conditionnel est, comme toujours, à l'origine de bien des angoisses. Les aléas d'une agression sont passés en revue : nervosité fatale d'un protagoniste, l'arrivée inopportune d'un témoin ou de la police, présence d'alcool ou de drogue, une caisse qu'on ne réussit pas à ouvrir.

Enfin, la peur des représailles est présente dans tous les types de victimisation, que ce soit au moment d'une convocation devant le tribunal,

Mon garçon était très nerveux quand ils lui ont demandé d'aller à la cour. Puis ça faisait tout près de deux mois que ça s'était passé. Disons que mon garçon, il ne s'est pas rendu, parce qu'il avait peur des jeunes qui le reconnaissent puis qu'ils disent : c'est toi qui nous a dénoncés, ça fait que tu vas manger la volée. C'est pour ça qu'il ne s'est pas rendu. 
d'une requête pour identifier les suspects,

J'ai été énervé pendant un bout de temps. Surtout quand il a fallu que j'aille les identifier. Tu sais, c'était la première fois, ça m'a énervé, surtout qu'ils peuvent t'en vouloir après. C'est un gros stress et surtout il faut être sûr de ne pas faire d'erreur sur la personne.

ou de l'appel à la police,

Ils m'ont dit : ouvre pas ta trappe à personne sinon... Puis ils m'ont décrit ce qu'ils allaient me faire et à mon petit-fils si je parlais. J'étais terrifiée, je ne sortais pas, je dormais assise sur un fauteuil, j'avais peur de me coucher, les lumières allumées toujours. Je ne répondais pas au téléphone. Puis, au bout de quinze jours, j'ai appelé mon fils et il a appelé la police.

Il arrive souvent que les craintes soient moins bien identifiées ou plus généralisées. La peur est alors liée à une catégorie de situations, un type de personnes, certaines circonstances ou encore, elle n'a aucun objet spécifique et se vit comme une angoisse diffuse. Ainsi, les femmes victimes d'agressions sexuelles ou de violences de la part de leur conjoint tendent à développer une méfiance à l'égard de tous les hommes. Cette constatation est corroborée par plusieurs études quantitatives (Burgess 1974, Katz et Mazur 1979). Les propriétaires et employés de "dépanneurs " commencent à ressentir de l'anxiété lorsque le soir arrive. Telle autre personne, victimisée au travail, ne peut plus occuper son emploi ou un emploi similaire. Une autre panique à l'idée de devoir monter dans un autobus ou un train de métro. Des personnes agressées chez-elles durant la nuit auront des angoisses à l'heure du sommeil, sans que ces angoisses soient axées nécessairement sur la crainte que l'événement se reproduise.

Ayant été agressée par un jeune homme "ordinaire ", bien mis, C'est l'arme qui m'a fait peur, pas le jeune homme, parce que le jeune homme, il avait l'air bien propre, assez que j'aurais jamais cru qu'il avait une arme. C'était un jeune homme qui était bien mis, un jeune homme tout jeune, un petit gars de 17 ans.

une vendeuse a eu peur de tous les jeunes durant des mois,

Quelqu'un entrait, un jeune, je m'imaginais toujours qu'il avait un gun dans les mains. J'avais peur de tous les jeunes qui rentraient. La réaction sur moi, c'est j'avais peur des jeunes. Comprenez-vous ? Puis là, j'ai tombé malade et je suis sur les soins des médecins par rapport à ça.

Il arrive aussi que l'objet et les circonstances des appréhensions soient tout à fait étrangers à la situation qui a été vécue. Ainsi, une femme agressée chez-elle se sentait complètement en sécurité à la mai- 
son mais incapable de sortir seule. Dans certains cas, ce sont les situations nouvelles qu'on redoute même si elles ne comportent aucun risque de victimisation.

Nos résultats nous portent à croire que c'est dans les cas d'agressions physiques (à caractère sexuel ou non) que la peur est généralisée plutôt qu'axée sur un objet particulier comme il se produit le plus souvent pour les victimes de vol qualifié.

Et comment se manifeste cette peur ? Ses expressions varient naturellement selon qu'elle est généralisée ou spécifique. Il peut s'agir d'une tension ressentie dans certaines circonstances.

Ça devient une pression terrible. On vit sous tension tout le temps qu'on travaille, à se demander si c'est pour aujourd'hui ou pour une autre fois.

D'autres victimes nous ont confié qu'elles avaient peur continuellement où qu'elles soient. Elles éprouvent une angoisse diffuse, une inquiétude qui n'a pas de motif précis.

Plusieurs personnes qui ont subi un cambriolage ont peur d'entrer chez elles seules. Elles écoutent à la porte, à l'affût de bruits insolites, avant de franchir le seuil, puis elles font le tour des pièces pour vérifier si quelqu'un ne s'est pas introduit en leur absence. Un commerçant, victime de plusieurs vols à main armée, suspectait tous ses clients et appelait sans cesse la police. Tel autre a vendu son magasin après un premier vol.

On a vendu. Le lendemain matin, mon mari a mis l'annonce sur la presse, puis ça s'est fait le lendemain matin. Penses-tu que mon mari était pour se faire tirer ?

Tel autre a également vendu son magasin.

J'avais tellement peur que le gars revienne régler mon compte que, après le hold up, j'étais plus capable de rester toute seule. Mon mari a dû perdre six mois d'ouvrage pour rester avec moi. Puis on a dû tout liquider la marchandise puis vendre. J'avais trop peur.

Des employés laissent leur emploi. Des gens déménagent, soit parce qu'ils ont été agressés à domicile, soit parce qu'ils ont peur que leurs agresseurs essaient de les retrouver et de leur faire un mauvais parti.

On a vendu notre chalet, notre restaurant, notre maison. On a aussi changé notre numéro de téléphone pour pas qu'il nous retrace. 
Les cauchemars sont assez fréquents comme les difficultés à trouver le sommeil. Ces sentiments de terreur ne font pas irruption seulement la nuit. Un certain nombre de personnes sont devenues méfiantes à l'égard des inconnus, les percevant comme des agresseurs potentiels. Certaines victimes d'agressions physiques, sexuelles ou autres, sont devenues incapables de rester seules ; une femme s'est même mariée pour cette raison, après une victimisation. D'autres personnes traversent la rue chaque fois qu'elles entendent des pas derrière elles. Enfin, on a peur des espaces ouverts ou des ascenseurs, des lieux publics ou des rues désertes, des inconnus, des hommes, des expériences nouvelles, de tout quoi.

C'est une crainte qui est là. Je suis demeurée craintive, très craintive, le soir. Je vais barrer tous les châssis, je vais barrer les portes, le soir, parce que j'ai peur. Le premier craquement, je saute. Parce que je sais jamais s'ils reviendront ou s'ils vont rester tranquilles. Après l'expérience que j'ai eue... C'est dur, vous savez, de vivre dans une crainte continuelle.

On aura réalisé, à ce point, que la peur varie chez les sujets aussi bien en intensité qu'en durée. On connaît encore très mal les facteurs associés à l'apparition de divers traumatismes chez les victimes (Fields 1980). Le sexe, l'âge, le fait de vivre seul ou non, les expériences antérieures d'événements traumatisants, la durée et la gravité de l'acte subi, la situation sociale et psychologique de la victime au moment de l'agression, seraient autant d'éléments qui permettraient de prédire les séquelles de la victimisation.

Comme notre échantillon n'est pas représentatif et que, de plus, aucune évaluation psychologique n'a été faite, nous ne sommes pas en mesure de nous prononcer sur les raisons pour lesquelles plusieurs victimes continuent à avoir peur longtemps après l'événement subi alors que certaines l'oublient rapidement. Il semble que les hommes, surtout les jeunes, récupèrent plus facilement que les femmes, que le viol et la tentative de meurtre soient les actes les plus terrorisants, et que la façon dont le crime a été perpétré influence les réactions à long terme (présence d'arme, nombre d'agresseurs, déguisement, isolation de la victime). L'influence du nombre de victimisation antérieures est plutôt brouillée. Dans certains cas, les effets d'une première victimisation s'estompent rapidement.

J'ai pour mon dire que, si j'ai vécu 58 ans sans me faire attaquer... Non, j'ai pas peur, c'est une chose qui arrive. Là, je vais être correct pour un autre 58 ans. 
On voit aussi des commerçants et des employés d'institutions financières qui ont connu nombre de vols à main armée avant de flancher au nième. Par contre, d'autres s'y habituent.

Après le premier, je pensais à laisser la banque. J'avais tellement peur. Je surveillais les clients tout le temps. C'est le deuxième qui m'a remise d'aplomb. Je l'ai bien pris. Là ça ne me dérange plus. Y a rien là. Ça fait partie du métier.

Souhaitons que cette attitude dure tout le reste de sa carrière!

\section{DES MESURES DE PROTECTION ACCRUES}

Tous les sujets interviewés, sauf les employés de banque, ont adopté des mesures de prévention suite à leur expérience. Dans la majorité des cas, surtout s'il y avait eu vol ou tentative de vol, ces mesures comportaient l'achat, l'installation et l'utilisation de dispositifs de sécurité (serrures, verrous, chaînes, système d'alarme, clôtures...). Certains ont consulté des policiers ou des spécialistes en prévention.

Plusieurs commerçants ont changé la disposition des lieux (installer la caisse plus près d'une fenêtre par exemple), ont commencé à faire des dépôts bancaires plusieurs fois par jour, ont acheté un chien. Les précautions qu'ils peuvent se procurer seraient toutefois assez minimes à cause des coûts financiers et sociaux relativement élevés.

Qu'est-ce que tu veux, quand le gars vient puis qu'il te pointe le fusil dans la face, tu peux pas faire grand chose. La seule chose que $\mathrm{j}$ 'ai faite, le système d'alarme c'est bien dispendieux pour nous autres, c'est comme le détective me le disait, mettre mon argent dans deux caisses, c'est enlever l'argent à mesure. On refuse de changer les billets, on aime mieux perdre la vente. Ça paraît simple mais c'est compliqué. En tous cas, comme ça, ils.te volent pas grand chose, les bandits, et ils sont pas généralement intéressés à revenir.

Ainsi, malgré les inconvénients qu'il comporte, le moyen le plus généralement utilisé serait de conserver peu d'argent en caisse, ce qui peut être une protection contre les pertes élevées mais pas nécessairement contre l'agression elle-même. Les commerçants peuvent aussi garder les portes fermées à clé et n'ouvrir qu'aux clients connus ou qui paraissent honnêtes.

Avec mon buzzer, je n'ai pas de problèmes. Les magasins devraient tous s'arranger comme nous autres. Quand mon mari n'est pas là, je regarde qui c'est, puis s'il a l'air louche, je n'ouvre pas. Je perds peut-être des ventes mais c'est pour protéger notre vie. 
Selon certains, il ne faut pas aller trop loin pour se protéger car, alors, on risque davantage. S'il n'y a pas suffisamment d'argent dans le tiroir, le voleur frustré peut devenir violent. Un système d'alarme pourrait effrayer un intrus qui, dans sa panique, blesserait ou tuerait des gens. Si les brigands n'ont pas accès assez facilement à la caisse, ils risquent de tout casser. Des employés d'institutions financières se prononcent contre certaines mesures telles les vitres anti-balles parce qu'ils craignent les prises d'otages, un crime qui les terrorise davantage que les vols à main armée. Selon deux d'entre eux, les sonnettes d'alarme sont non seulement inutiles mais dangereuses.

Enfin, dans certains établissements, on accepte la protection organisée offerte par la pègre locale. Au dire d'un interlocuteur, ce serait la sécurité la plus efficace, la moins coûteuse.

Les particuliers ont tendance à changer ou à restreindre leurs activités, évitant toute une situation qui apparaît dangereuse. Si on continue à sortir seul le soir, on voyage en taxi, à moins que la victimisation ne soit associée à une voiture de taxi, en quel cas on privilégiera les transports en commun. Des victimes d'agressions sexuelles ont suivi des cours d'auto-défense. Certaines d'entre elles transportent divers objets qui pourraient servir à contre-attaquer. Nous avons déjà vu que des personnes déménagent, changent d'emploi, obtiennent un numéro de téléphone confidentiel, vont vivre chez des amis ou se marient. Dans des cas extrêmes, on se terre chez soi, abandonnant son emploi et toute activité sociale.

J'ai vécu comme une bête en cage durant deux ans. Après être sorti de l'hôpital, j'ai pris un autre logement et je suis resté caché là sans parler à personne. J'avais peur de tout. Je ne suis pas rẹtourné travailler, je ne sortais jamais. À part l'épicerie, je n'allais nulle part et ne ne voyais personne.

De telles réactions d'isolement, loin de protéger, augmentent les risques d'agression. Et voilà deux caractéristiques de la peur : son irrationalité et la vulnérabilité qu'elle provoque. Les mesures de prévention adoptées ne sont pas toutes de nature à protéger, bien au contraire, ou encore elles peuvent jouer dans un sens comme dans l'autre. Bien des sujets en sont conscients. Telles ces personnes qui laissent toujours de l'argent dans un endroit facile à repérer afin que les voleurs ne vandalisent pas la maison dans leur recherche du butin. Telles autres refusent de demander à des voisins ou à la police de surveiller leur maison en leur absence : le moins de gens seront au courant de leur départ, le moins élevés les risques de cambriolage. 
Nous avons observé deux situations où les victimes ont tendance à ne pas adopter les mesures les plus logiques pour prévenir une nouvelle agression. Les femmes qui se sont fait arracher leur sac ont modifié leurs habitudes de sorties et restreint leurs activités mais elles continuent à porter un sac même si elles n'y déposent plus leur argent ou objets précieux. Le cas des femmes violentées par un conjoint est plus complexe. Le départ du foyer semble la solution la plus rationnelle mais c'est généralement la dernière à laquelle on a recours. La situation financière, le problème des enfants, l'ambivalence des sentiments à l'égard du conjoint, rendent cette mesure extrêmement coûteuse psychologiquement, socialement et économiquement.

Enfin, certains moyens de prévention suggérés par la police, les agents de sécurité, les amis, la publicité anti-crime, semblent trop contraignantes pour qu'on puisse se résoudre à les adopter.

J'ai un pamphlet, une petite brochure qu'ils m'ont donné et qui indique tout ce qu'il faut faire et ne pas faire. Trois feuilles imprimées des deux côtés. Je pourrais bien l'apprendre par cœur. Mais si je fais tout cela, ce n'est plus la peine de vivre. C'est comme s'il fallait que les gens qui choisissent d'être violents, eux, il faudrait leur laisser toute leur liberté, c'est comme si le crime était permis et que c'est les gens qui ne veulent pas de mal à personne qui doivent se priver de toute liberté.

Il n'est pas très surprenant que les sondages ne découvrent pas, chez les victimes, des corrélations aussi élevées entre la peur du crime et la prévention que le sens commun peut suggérer. Les mesures de prévention utilisées sont si variées et parfois irrationnelles, qu'on ne saurait induire de l'absence de mesures élémentaires ou de crainte de sortir seul le soir, l'inexistence de peur.

\section{DES VICTIMES TOLÉRANTES OU PUNITIVES ?}

Pour se protéger contre le crime, des victimes se procurent une arme, ou songent à le faire. Non seulement les commerçants mais aussi les particuliers ont considéré cette éventualité ou ont déjà passé aux actes, qu'il s'agisse d'une arme à feu, d'un couteau ou d'une aiguille à tricoter.

Chez les commerçants, l'arme peut être un moyen d'intimidation dont on n'a pas l'intention de se servir.

Ça se parle beaucoup qu'on a une arme ici. Ils se le disesnt de l'un à l'autre. On est bien connus dans le coin. Depuis que ça se sait qu'on est armé ça a diminué les vols. Ils en ont entendu parler beaucoup puis ils ont dit : c'est pas bon d'aller là. On s'en ser- 
virait ou pas, c'est une autre affaire. En tous cas, on se sent plus fort.

L'arme peut aussi être vue comme un élément qui augmente le danger.

Bien, je ne le dis pas qu'il est armé parce que si ça se sait, mais qu'ils viennent, ils vont tirer en entrant.

Par contre, certains expriment la ferme intention de s'en servir à la première occasion.

Les policiers m'ont dit : tirez dessus, c'est la seule chose à faire avec des pourris comme ça. Si j'ai la chance, je tire avant qu'ils tirent. J'attendrais de voir l'arme mais je ne poserai pas de question. Je le descendrais et j'hésiterais pas une seconde.

Des particuliers, des femmes aussi bien que des hommes, se proposent de se défendre violemment à l'avenir en cas d'attaque.

Moi, je vais aller pour les yeux. Ça fait mal. Ça incapacite. J'aurais pas la moindre hésitation ou la moindre pitié. S'il est assez animal, si quelqu'un agit comme un animal sauvage avec moi, je vais le traiter en animal sauvage.

De tels propos ne rassurent guère sur le climat d'une société, sur la qualité de la vie qui y règne. Serait-on revenu à un régime primitif de vengeance? Il faut d'abord noter que très peu de victimes, même agressées à plusieurs reprises, ont actualisé leurs menaces. Il faut ensuite remarquer que la contre-attaque est envisagée comme un moyen de prévention beaucoup plus que comme une forme de représailles.

On n'a aucune protection, qu'est-ce que vous voulez que je vous dise ? La police, ça vaut rien. Les bandits rient d'eux autres puis de nous autres. Ils défendent aux honnêtes gens le port d'armes mais ils laissent les bandits s'armer jusqu'aux dents. Le mieux, c'est d'avoir une M1, c'est ridicule mais ça c'est permis. Mais quand tu vas à Parthenais, même quand tu dis que ta vie est menacée, ils te refusent le permis. Alors, on va-tu attendre d'être étendu à la morgue ou qu'ils aient tué notre femme ou nos enfants?

Beaucoup plus que l'intention de se défendre violemment, les opinions concernant les sentences appropriées à divers types de criminels ou à leurs agresseurs, témoignent du degré de punitivité des victimes. En effet, il s'agit maintenant des mesures envisagées après l'acte, une fois que leurs vies ou leurs biens sont hors de danger immédiat.

Concernant l'action policière et les sentences rend'ses par les tribunaux, les victimes diffèrent peu de leurs concitoyen: Les policiers ne sont pas assez efficaces dans la recherche des criminels et leur action est entravée par la cour, dit-on. Les vrais criminels échappent 
trop facilement à la justice alors qe les petits délinquants sont sévèrement punis. La prison n'est pas une solution efficace mais on n'y connaît pas d'alternatives. Les libérations conditionnelles vont à l'encontre de toutes les tentatives pour enrayer la criminalité.

Le groupe de recherche sur les attitudes envers la criminalité (G.R.A.C. 1982) faisait remarquer l'absence totale de rapprochement entre la punitivité, la victimisation, le sentiment de sécurité, et la peur de victimisation. Il soulignait que ses propres résultats appuyaient les conclusions de Stinchcombe et al (1980), à savoir que :

- premièrement, les femmes, qui sont moins victimes de crimes violents, ont plus peur mais ne sont pas plus punitives que le reste de la population ;

- deuxièmement, que les individus qui ont été victimisés n'ont pas plus tendance que les autres à favoriser des mesures plus punitives.

Nos propres résultats, obtenus à l'aide d'une méthodologie différente, convergent dans ce sens. Toutefois, lorsqu'il s'agit de leurs propres agresseurs, les victimes semblent plus tolérantes qu'elles ne le sont pour les criminels en général. Il n'est pas rare d'obtenir des commentaires comme :

Moi, je trouve ça bien d'valeur pour le jeune et puis pour ses parents. J'ai bien de la pitié pour lui. J'ai prié pour lui, vous savez. Il était jeune, tu vois. Il n'avait que 17 ans. Si ça avait été le gros mec, un homme de 40 ans, qui va te foutre des coups ou te prendre en otage, j'aurais peut-être eu une autre attitude. Mais lui,je voulais lui donner une chance. J'ose espérer qu'il n'a pas été incarcéré pour une affaire de vol.

Lorsque leur agresseur n'a pas été retrouvé, n'a pas reçu de sentence, ou lorsque la sentence est inconnue, les victimes ont du mal à exprimer leurs souhaits concernant les mesures à adopter à l'endroit des criminels. Leurs opinions sont alors passablement stéréotypées. Il est plus facile d'exprimer de la satisfaction ou de l'insatisfaction face à une mesure précise dans un cas spécifique.

Des propos des victimes se dégagent quatre grandes tendances. Les deux plus fréquentes touchent la réclusion et la correction. Plus rares sont la réparation et le pardon.

Si l'acte a été vécu comme particulièrement sérieux, et comme une atteinte à l'intégrité personnelle, si l'agresseur est considéré malade mental, sadique, ou enraciné dans le vice, la seule réaction appropriée semble être la réclusion à perpétuité ou presque. Qu'on le mette hors d'état de nuire, pour longtemps, pour toujours, puisqu'il n'y a au- 
cun espoir d'amélioration. Qu'il ne puisse surtout pas sortir et commettre de nouvelles agressions.

En théorie, je suis contre la prison ou l'hôpital psychiatrique. Mais, pour un cas comme le mien, tu vois, il n'y a pas de choix. Moi, je ne pourrai jamais respirer s'il est au large. C'est lui ou moi. Si lui est dehors, moi je suis en dedans. Sans compter toutes les autres qu'il a détruites, tu sais, ou bien la prochaine fois il va tuer, il n'a plus grand chose à perdre. Non, non, il n'y a pas de voie il faut l'enfermer et surtout pas le laisser sortir. Moi, je ne vois pas d'autre solution, j'ai beau chercher. Je ne veux pas qu'il sorte jamais.

Les proches des victimes d'homicide semblent se distinguer en ce qu'ils ne ressentent pas de crainte pour eux-mêmes mais plutôt pour des victimes potentielles. Malgré la gravité du crime, ils expriment une certaine démission parce que leur perte est irrémédiable et aucun châtiment ne pourra jamais la compenser. Bien sûr, on devrait mettre le criminel hors d'état de nuire, mais c'est dit sans passion.

Par "correction", les victimes entendent toute action qui vise à intimider le criminel, à l'obliger à réfléchir aux conséquences de ses actes, à changer d'orientation. On l'estime particulièrement efficace chez les jeunes et pour des premiers délits.

En les prenant jeunes, si on leur donne une bonne correction, je sais bien qu'ils ne recommenceront pas. La police l'a pris puis ils lui ont donné une bonne leçon. Ils l'ont amené au poste puis ils lui ont dit : pour cette fois, tu n'as pas de dossier, tu n'as absolument rien mais si tu viens à te faire prendre une autre fois, on te donnera pas de chance. Moi, j'ai trouvé ça très bien, c'était une bonne leçon, de première classe.

La correction, c'est également faire vivre à l'agresseur la peine qu'il a infligée et lui faire connaître clairement qu'il a eu tort, que des personnes ont été lésées par ses actions et qu'il s'est attiré la réprobation générale.

Les petits culs en avant trouvaient ça bien drôle. Ils se moquaient de moi. Je ne vois pas pourquoi ils se seraient privés. C'est eux que tout le monde défendait. Moi, j'étais toute seule. (...) Moi je pense que s'il y avait eu une seule personne, une seule, le juge, ou l'avocat, ou n'importe qui, qui m'avait dit : c'est vrai, madame, vous avez subi un crime, $c$ 'était injuste, bien, je vais aller loin là, mais je leur aurais peut-être pardonné. $\mathbf{C}^{r}$ mme c'est là, c'était à moi à me défendre et tu entres dans ce jeu-ià et je t'assure que plus ça allait dans le procès, plus je voulais qu'ils en mangent. 
Le sentiment d'injustice subie est très grand chez les victimes et il est exacerbé par le traitement reçu du système de justice criminelle. On voudrait être compris même de l'agresseur.

Une sentence comme exactement ce qu'ils ont fait. Davantage calculée, davantage recherchée. Pour qu'ils comprennent la portée de leurs gestes et ce que, nous, on souffre.

Ce souhait revient assez fréquemment : qu'on fasse subir au criminel la souffrance qu'il a infligée, ni plus, ni moins, de sorte qu'il comprenne. ceil pour cil!

La correction suppose donc que le délinquant peut s'amender mais, en contrepartie, il ne changera son comportement que s'il est puni ou traité :

J'aurais aimé ça que les gars aient au moins une charge contre eux autres. Qu'ils s'en souviennent de cette affaire là. Ils vont recommencer sur d'autres. Puis quand est-ce qu'on va les pogner ? Ça commence par un vol, ça finit par un meurtre, une affaire comme ça. Tandis que là, s'ils les auraient punis, je le sais pas, un mois de prison, une affaire comme, ils peuvent réfléchir un peu. Après avoir réfléchi, le gars, il aurait peut-être pas recommencé une deuxième fois.

Il est rare que les victimes suggèrent spontanément des mesures de réparation par l'agresseur. Une victime de vol qualifié a proposé :

Il y aurait les dédommagement par l'agresseur ou sa famille. En réalité, comme clause constructive, je ne m'y suis pas beaucoup arrêtée. Comment améliorer le système? Je ne sais pas comment y remédier. Je pense que c'est un problème en partie social, en partie d'éducation dans la famille. Le dédommagement ce serait une éducation à faire en même temps que ce serait une compensation, un soulagement pour nos pertes.

Une autre personne qui avait aussi subi un vol à main armée dans son établissement aurait aimé que le juge impose une mesure de restitution au voleur. Au lieu ou en sus de la détention?

C'est pas que je suis contre l'incarcération, mais disons, il y était susceptible, mais il aurait dû être obligé de faire la restitution. Admettons qu'il a volé $100 \$$, on a récupéré $70 \$$, les sept dixième, alors il aurait dû être responsable du trente pour cent qui reste.

Elle poursuit en suggérant qu'on instaure des programmes de travail où les personnes condamnées et qui sont sans emploi pourraient être suffisamment rémunérées pour faire réparation à leur vic- 
time. Cette idée est reprise et développée avec quelques variantes par une personne qui a subi un cambriolage.

Dans le cas du vol par effraction que j'ai raconté, les jeunes qui sont impliqués, ça fait dèjà deux ans de ça, ces jeunes, leur sentence, c'était un couvre-feu. Je ne dis pas qu'ils auraient dû être en prison mais je dis qu'ils aurient dû être responsables de faire une restitution après le vol. Il y avait peut-être $500 \$$ de volés ou plus, et je n'étais pas couvert et ils n'ont pas fait la restitution. Même si ça leur prend dix ans ou qu'ils paient dix piastres par quinze jours, mais c'est une dette qui devrait être honorée.

On pense aussi à la réparation symbolique, à de tierces personnes, surtout dans les cas où il n'y a pas eu de vol ou de vandalisme. Le criminel devrait prendre soin des malades, des personnes âgées, faire des bonnes œuvres... Un homme, handicapé de façon permanente à la suite d'un crime, suggère que les auteurs de voies de fait soient condamnés à servir dans des centres de réadaptation physique.

Lorsque c'est l'interviewer qui suggère la réparation, les réactions sont mitigées, prudentes. Oui, peut-être, à condition que, si, seulement dans tel ou tel cas et, surtout, qu'il n'y ait pas de contact entre agresseur et victime. Le plus souvent, les sujets d'entrevue n'avaient jamais auparavant réfléchi à ce type de solution.

Conditionnel, le pardon ne s'applique qu'aux jeunes et aux premières offenses. Il prend la forme de la prière ou de vœux pieux ; plus concrètement, il se traduit en velléité d'un retrait de plainte, en un refus d'identifier le suspect ou même par l'expression de tendre la main au coupable.

J'aurais absolument pas voulu qu'ils le mettent en prison. Sans être paternaliste, ce jeune-là, c'était absolument clair que ses parents c'étaient deux ivrognes et qu'ils ne pouvaient pas l'aider. Ce jeune-là, je me suis dit que je pouvais peut-être l'aider. Le juge, je me rappelle que je me suis dit que si le juge voulait établir un dialogue avec moi, j'étais prêt à faire quelque chose, je ne sais pas trop quoi, lui donner une job à temps partiel.

Seulement deux des victimes rencontrées avaient été sollicitées pour s'engager dans un processus de conciliation. Une d'entre elles a carrément refusé, disant :

Il y a bien une sainte limite. Après tout le trouble qu'il m'a causé, faudrait que je l'aide encore?

L'autre a accepté "par charité chrétienne" et en retient l'impression que

Le système les cajole, c'est incroyable. Pauvre petit, accepteraistu de faire ça? Non? D'accord, faut pas t'offusquer, mon petit. 
Et vous, monsieur, faut le comprendre. On a fait quatre séances comme ça avec le petit garçon gâté. Puis j'ai dit oui à n'importe quoi pour en finir. C'est pas sérieux.

Une autre victime aurait aimé connaître ses agresseurs, les rencontrer, quand elle a appris qu'ils étaient des mineurs et non de dangereux bandits.

De penser que quelqu'un était dans ma maison, ça fait drôle. Ça donne la chair de poule. Dans mon esprit, je m'imaginais des bandits de plus de six pieds, 200 livres, tatoués, avec des pistolets, des chaînes, des masques. J'ai appris que c'étaient des adolescents. J'aurais aimé ça les voir, je pense que j'aurais eu moins peur par la suite.

Enfin, quand les agressions se sont produites entre personnes qui se connaissaient, une forme de pardon est souvent préconisée.

En somme, à l'égard des criminels en général, l'attitude des victimes est semblable à celle des autres citoyens, assez répressive, assez stéréotypée. Par contre, même si certains de leurs propos peuvent paraître plutôt cruels et barbares, les victimes ont tendance à faire preuve de tolérance à l'égard de leurs agresseurs. Leurs opinions sont plus nuancées, les sentences adaptées aux situations perçues, individualisées. Elles sont motivées surtout par le désir de se sentir personnellement en sécurité ou de protéger d'autres personnes, et parfois par un besoin de justice ou d'humanité.

\section{MESURER LA PEUR CHEZ LES VICTIMES}

Plusieurs auteurs ont déjà remarqué qu'il est difficile de définir le concept "peur du crime ". Des progrès ont été faits en ce sens. Ainsi, la distinction entre peur concrète et peur diffuse (Figgie 1980, Brillon et al., 1982) clarifie déjà la notion de peur. On observe d'ailleurs, dans les sondages, une relation entre peur concrète et expérience de victimisation, relation qui est quasi inexistante si la distinction n'est pas faite. Nul doute que chez les victimes la peur est très concrète; les propos cités sont éloquents.

Les manifestations de la peur varient énormément comme sont également multiples les moyens qu'on prend pour se protéger. On pourrait penser que les victimes sont beaucoup plus imaginatives et originales sur ces plans que le reste de la population. Or les mesures développées ne tiennent pas compte du caractère varié et atypique de la peur et de la prévention. Il est évident que si on ne mesure la peur que par un indice, éviter de sortir seul le soir, nos sujets d'entrevue ne 
se distingueront pas des autres personnes. Enfin, il faut tenir compte des situations, de l'objet des craintes et des ressources dont disposent les gens.

Dans presque tous les sondages où on a étudié la relation entre la peur et la victimisation, cette dernière variable se référait aux expériences des derniers six ou douze mois. Or, la peur persiste assez souvent durant des années. Aucun indice de gravité n'a été élaboré. Certains crimes particulièrement sérieux, mais peu fréquents, ont été éliminés (meurtre, enlèvements). La victimisation a donc été mesurée imparfaitement et les groupes " victimes" et "non victimes " n'étaient pas purs. D'ailleurs, la notion même de victime reste à définir.

Enfin, au chapitre de la punitivité, les discours des victimes font ressortir le besoin de connaître les motifs des sentences préconisées, comme la nécessité de distinguer entre les attitudes envers le crime en général et les mesures souhaitées dans des cas particuliers. Puisque tant de législations ont été présentées et adoptées au nom des victimes, pour leur profit, il serait grand temps de connaître ce que les victimes désirent et pourquoi. Il n'est pas certain que nous découvrirons une correspondance entre leurs besoins et les politiques criminelles existantes ou proposées.

\section{BIBLIOGRAPHIE}

BARIL, M. (1977) : L'image de la violence au Québec, Rapport $\mathrm{n}^{\circ}$ 4, Montréal, C.I.C.C., G.R.A.C.

BURGESS, A.W. et HOLMSTROM, L.L. (1974) : Rape : Victims of Crisis, Bowie, MD, Brady.

EITINGER, L. (1964) : Concentration Camp Survivors in Nonway and Israel, London, Allen \& Unwin.

FIELDS, R.M. (1980) : "Victims of Terrorism : The Effects of Prolonged Stress ", in Evaluation for Change, Special Issue, p. 76-84.

FIGGIE REPORT (1980) : America Afraid, The Figgie Report on Fear of Crime, Part I, The General Public, A-T-O Inc. Ohio.

GAROFALO, J. (1979) : "Victimization and the Fear of Crime", Journal of Crime and Delinquency, 16, p. 80-97.

GENN, H.G. (1976) : " Findings of a Pilot Survey of Victimization in England ", Victimology, 1-2, p. 253-262.

GRENIER, H. et MANSEAU, H. (1977) : En quête de justice : les petits commerçants victimes de vol à main armée, Montréal, C.I.C.C., G.R.A.C.

HINDELANG, M.J., GOTTREDSON, M.R. et GAROFALO, J. (1978) : Victims of Personal Crime : An Empirical Foundation for a Theory of Personal Victimization, Cambridge, Mass., Ballinger.

KILLPATRICK, D.G., RESICK, P.A. et VERONEN, L.J. (1981) : "Effects of a Rape Experience : a Longitudinal Study ", Journal of Social Issues, 37, 4, p. 105-122. 
KATZ, S. et MAZUR, M.A. (1979) : Understanding the Rape Victim : A Synthesis of Research Findings, New York, Wiley.

RIGIER, S. (sous presse) : "The Impact of Crime on Women ", in Lewis, D.A. (ed) Reactions to Crime, Beverly Hills, Sage.

SKOGAN, W.G. et MAXFIELD, M.G. (1981) : Coping with Crime : Victimization, Fear, and Reactions to Crime in Three American Cities, Beverley Hills, Sage.

VAN DIJK J.J.M. (1978) : " Public Attitudes toward Crime in the Netherlands", Victimology, 3-4, p. 265-273. 